\title{
Development of a Reusable Atomic Oxygen Sensor using Zinc Oxide Thick Films
}

\author{
Juan Carlos Valer, Graham Roberts, Alan Chambers, John Owen, Matthew Roberts
}

\begin{abstract}
The paper describes the fabrication and testing of thick film zinc oxide sensors that could be used to monitor the atomic oxygen fluence experienced by spacecraft in low Earth orbit, or that produced in ground-based atomic oxygen sources. Impedance spectroscopy measurements were carried out on the sensors to determine the conduction mechanisms within the thick films when exposed to atomic oxygen. The results indicate that the sensors' total resistance increases linearly with time when they are exposed to a constant flux of atomic oxygen, and that the sensors can be regenerated by heating.
\end{abstract}

Index Terms-Atomic Oxygen, Space Environment, Thick film sensors, Zinc Oxide.

\section{INTRODUCTION}

Atomic oxygen (AO) is the main atmospheric constituent in Low Earth Orbit (LEO) at altitudes between 200-1000 km. Atomic oxygen is formed by the photo-dissociation of molecular oxygen by solar ultra-violet (UV) radiation [1]; at these altitudes the probability of recombination is low due to the low atmospheric density and hence the AO is a stable species. Despite its low density it is well known [2] that AO causes high levels of damage over time to many spacecraft materials, particularly those located on forward-facing (ram direction) surfaces. For example, silver electrical contacts are readily oxidised and become non-conducting whilst polymer thermal protection blankets may be eroded, causing their optical properties (emissivity and absorptivity) to change and thus affecting their performance. In both cases the damage processes are enhanced by the high collision energy (approx. 5 $\mathrm{eV}$ ) of the AO. The AO flux (usually expressed as atoms $\mathrm{cm}^{-2}$ $\mathrm{s}^{-1}$ ) is highly variable, depending primarily on altitude and level of solar activity [1].

As an example, for the International Space Station (ISS), residing in a roughly $330 \mathrm{~km}$ circular orbit, a typical AO flux

J.C. Valer was a doctoral student at the University of Southampton. His contact details are:1 Rowleys Mill, Uttoxeter New Road, Derby, DE22 3TJ, UK; valer.jc@gmail.com.

G Roberts, A Chambers, and J Owen are all with the University of Southampton, Southampton, Hants, SO17 1BJ, UK. G Roberts' e.mail is: G.T.Roberts@soton.ac.uk; his phone is +44(0) 238 059-2325.

Copyright (c) 2012 IEEE. Personal use of this material is permitted. However, permission to use this material for any other purposes must be obtained from the IEEE by sending a request to pubs-permissions@ieee.org experienced by a ram-facing surface at solar mean conditions is approximately $3 \times 10^{14}$ atoms $\mathrm{cm}^{-2} \mathrm{~s}^{-1}$. This flux is typically two orders of magnitude higher than that of the next most abundant atmospheric species at that altitude, which is molecular nitrogen. However, because of its variability, it is useful to have the means to monitor the time-integrated flux, or fluence (expressed in atoms $\mathrm{cm}^{-2}$ ), of $\mathrm{AO}$ that a spacecraft in a particular orbit receives, for example to determine the rate of degradation of materials or to plan adequate protection for the end-of-life performance of subsequent spacecraft that operate in a similar orbit. Many different types of sensor have been developed to measure the AO flux or fluence [3]; such sensors must be capable of operating under the high vacuum conditions prevalent in LEO and, ideally, at temperatures that typically might range from $-50^{\circ} \mathrm{C}$ to $+125^{\circ} \mathrm{C}$, although in practice some form of thermal control (active or passive) is usually employed to limit the operational temperatures to a much smaller range. Amongst these are sensors fabricated from thin films of silver [4] and carbon [5] which increase in resistance during exposure to $\mathrm{AO}$; however for these sensors the resistance change is irreversible and thus they exhibit limited lifetime. Sensors fabricated from metal oxide semiconductors such as zinc oxide $(\mathrm{ZnO})$ have also been developed to detect and measure atomic oxygen, as well as other planetary atmospheric species [6]-[8].

Past experience at the University of Southampton [9]-[11] with sensors fabricated using thin $(\sim 1 \mu \mathrm{m})$, sputtered films of zinc oxide has shown that atomic oxygen adsorbs to the surface of zinc oxide, an n-type semi-conductor material, with negligible diffusion into the bulk, removing some of the electronic density available for conduction and thereby increasing the resistance of the sensor; this change in resistance can be used to estimate atomic oxygen flux. The sensors were observed to saturate rather quickly, i.e. their resistance change became negligible beyond a certain AO fluence, shown to be approximately $10^{16}-10^{17}$ atoms $\mathrm{cm}^{-2}$ (equivalent to only a few minutes of exposure at ISS altitudes). However, unlike sensors constructed from thin films of silver and carbon, the resistance change was found to be reversible: heating the sensors resulted in the desorption of the adsorbed oxygen. The sensors were then observed to be responsive once again to the atomic oxygen flux, although significant hysteresis was noted in this regeneration process.

As the manufacturing route for these sensors was complex and relatively expensive, an alternative relatively simple and inexpensive sensor construction technique has been 
investigated with the aim of producing an $\mathrm{AO}$ sensor that is capable of regeneration.

\section{THICK FILM ZINC OXIDE SENSOR CONSTRUCTION}

As a natural evolution of our previous work with thin film sensors, and bearing in mind the required simplicity of construction, we chose to use screen-printed thick films [12] of $\mathrm{ZnO}$ as the basis for these new sensors. Thick films of $\mathrm{ZnO}$ have been used to detect organic vapours and other gases (e.g. [13]) but, as far as we are aware, have not previously been used for detection or measurement of AO. An inert alumina substrate was chosen, similar to that used by Osborne for his thin-film sensors [9], upon which an interdigitated array of gold contacts was screen-printed and fired. Subsequently, a layer of ink comprising $\mathrm{ZnO}$ powder in a-Terpineol (a natural solvent) was screen-printed over the gold contacts. The $\mathrm{ZnO}$ powder used was $99.9 \%$ pure commercial stock with a particle size of $<5 \mu \mathrm{m}$ (Sigma Aldrich product number 205532). After mixing with the solvent the resultant paste was mechanically milled to reduce the particle size and increase the homogeneity of the ink prior to screen-printing. Firing to a maximum temperature of $850^{\circ} \mathrm{C}$ followed; it is expected that this temperature is more than sufficient to eliminate all the solvent in the film. The typical thickness of the resultant $\mathrm{ZnO}$ layer was about $10 \mu \mathrm{m}$. The interdigitated geometry of the gold contacts reduces the effective length/width ratio of the $\mathrm{ZnO}$ resistance element, thus reducing the overall resistance of the sensor. This was necessary because the resistivity of the $\mathrm{ZnO}$ films produced was very high.

In total four sensors, approximately $9 \mathrm{~mm} \times 5 \mathrm{~mm}$ in size, were deposited on each alumina substrate. On the back of the alumina substrate a ruthenium oxide heater element, similar to that employed with the previous carbon and zinc oxide AO sensors [5], [9] was also screen-printed. This was subsequently used to control the sensor temperature during testing and also for regeneration heating.

It was also observed that the $\mathrm{ZnO}$ films deposited using the process described above were prone to mechanical abrasion and therefore required careful handling; some films were printed with a binder to improve their robustness and adhesion to the substrate [14] but, as the resistivity of these films was much higher (since the binder was non-conductive), resistance measurements were much more difficult and prone to error. Hence all the results presented in this paper concern the sensors fabricated from the pure $\mathrm{ZnO}$ powder without binder.

Microstructure analysis was carried out via Scanning Electronic Microscopy (SEM). It was found that the $\mathrm{ZnO}$ film comprised an agglomeration of crystallites of sizes ranging from 20-200 nm (Fig.1). Such a structure appears to be typical of thick films of $\mathrm{ZnO}$ prepared in this manner (e.g. [13]).

The sensors' electrical characteristics and response to atomic oxygen were investigated by measuring the overall (DC) resistance and by utilising impedance spectroscopy. It was hoped that the latter measurement technique would provide additional insight into the conduction mechanisms taking place within the $\mathrm{ZnO}$ thick films.

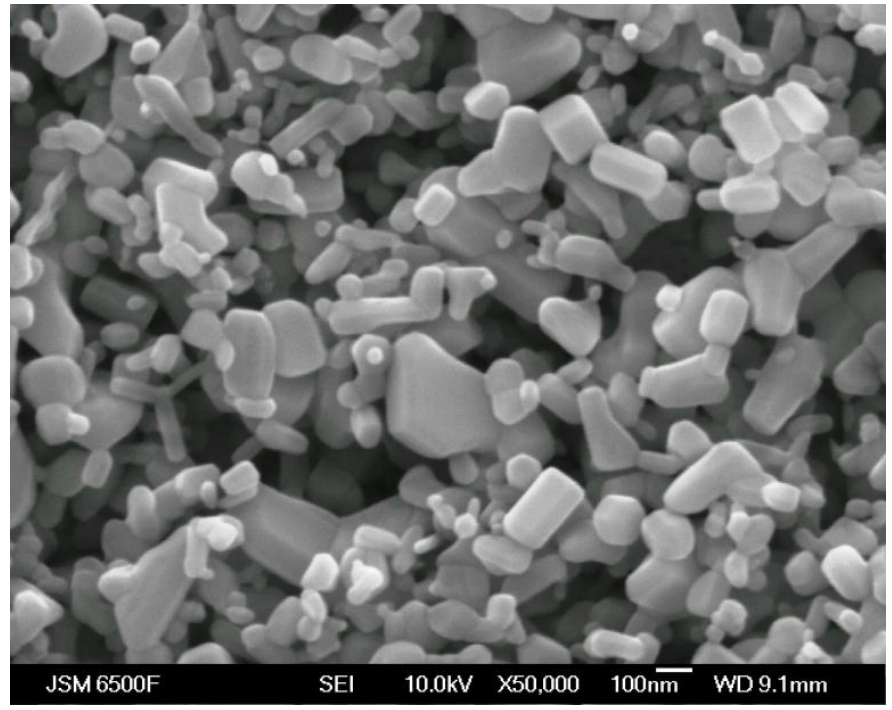

Fig. 1. Scanning electron micrograph (SEM) image of a typical $\mathrm{ZnO}$ thick film (magnification x50000).

\section{DC RESISTANCE EXPERIMENTS}

Before exposure to atomic oxygen a series of tests was carried out to determine the sensors' electrical characteristics under vacuum conditions. The sensors were loaded into a vacuum chamber and the pressure reduced to approximately 4 x $10^{-4} \mathrm{~Pa}$ using a turbo-molecular pumping system. Whilst pumping down the $\mathrm{DC}$ resistance $(\mathrm{R})$ of the sensors, estimated by recording the current flow for a given applied DC potential across the gold contacts, was monitored. It was observed that the resistance decreased by several (typically 3-5) orders of magnitude as the pressure was reduced. This effect was ascribed to the desorption of atmospheric species and was found to be reversible, in that the resistance increased by a similar amount when the vacuum chamber was brought up to atmospheric conditions again at the end of the experiment.

It was also found that non-ohmic behaviour (i.e. a nonlinear relationship between current and voltage) existed for applied potentials greater than $3.3 \mathrm{~V}$ and that the resistance would typically drop by an order of magnitude when the applied potential was increased from 5 to $50 \mathrm{~V}$. These observations are consistent with those of Ohashi et al. [15] for doped $\mathrm{ZnO}$ varistors, although in our case doping was not deliberately applied. All the following DC resistance results that are presented were obtained with applied potentials less than $3 \mathrm{~V}$ and hence in the ohmic (linear) range.

Once the sensors' resistance had stabilised under vacuum conditions, the effect of variations in sensor temperature was investigated. The sensor temperature was varied from $10^{\circ} \mathrm{C}$ to $70^{\circ} \mathrm{C}$, this being the range that a sensor with some form of temperature control might typically experience in service in space. A typical response is plotted in Fig. 2 and indicates that the natural logarithm of DC resistance increases linearly with the inverse of absolute temperature. Such a response is to be expected for a semiconductor material. The slope of the best fit straight line shown in Fig. 2 was used to obtain the conduction activation energy for the $\mathrm{ZnO}$ sensor. This was found to be $0.054 \mathrm{eV}$, which is similar to that found for $\mathrm{ZnO}$ thin films by Wortman et al. (0.047 eV) [16] and by Osborne 
$(0.039 \mathrm{eV})[9]$ and also, for $\mathrm{ZnO}$ single crystals, by Hagemark and Chacka $(0.043-0.045 \mathrm{eV})$ [17]. These results are all consistent with the donor level of interstitial zinc in $\mathrm{ZnO}$.

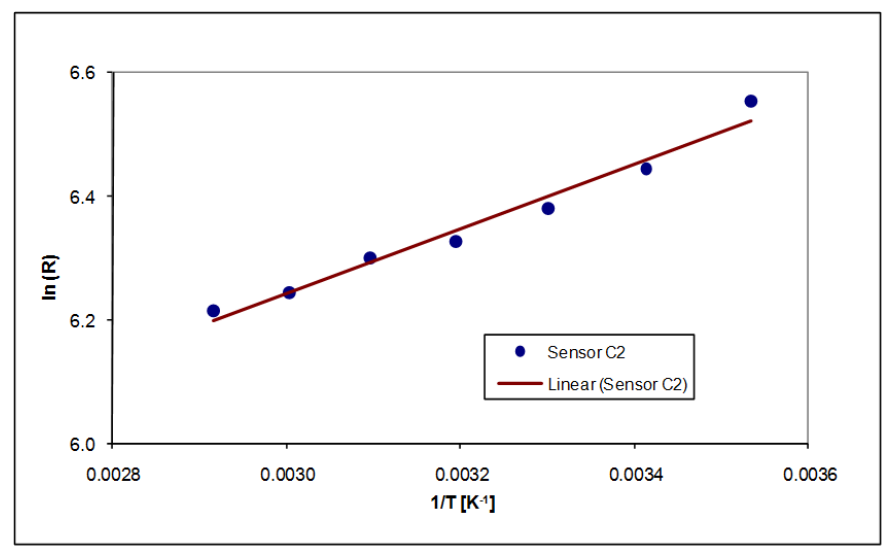

Fig. 2. Typical variation of the natural logarithm of DC resistance with inverse temperature for a $\mathrm{ZnO}$ thick film under vacuum conditions.

Several sensors were exposed to atomic oxygen in the ATOX test facility at the ESTEC establishment of the European Space Agency and the change in DC resistance was recorded during the exposures. The ATOX atomic oxygen source is of the pulsed laser-breakdown type, capable of producing a gaseous beam comprising predominantly neutral, ground-state and stable $\mathrm{AO}$ at time-averaged fluxes $\left(\mathrm{O}\left(10^{15}\right)\right.$ atoms. $\mathrm{cm}^{-2} . \mathrm{s}^{-1}$ ) and energies (approx. $5 \mathrm{eV}$ ) similar to those experienced by spacecraft in LEO [18]. The pulse rate in these experiments under ultra high vacuum was $1 \mathrm{~Hz}$; the sensor temperature during exposure was maintained at $35^{\circ} \mathrm{C}$.

During its operation the ATOX source also produces UV at a level that is several orders of magnitude higher than solar at LEO [19]. In order to isolate the effect of UV exposure on the sensors, some were covered with quartz windows; this material is transparent to UV radiation but resists the attack by AO. Some sensors were also blocked from the AO flux and the UV by alumina covers. Typical results are presented in Fig. 3. In this plot, resistances have been normalised with respect to the initial baseline resistance prior to $\mathrm{AO}$ exposure in order to illustrate more clearly the changes that took place.

It is observed that, after an initial transient when the AO exposure commences (at time $\mathrm{t}=0$ ) during which the resistance changes slightly, the resistances of the sensors covered with quartz and alumina did not change significantly, whereas that of the exposed sensor continued to increase as the total AO fluence increased with time. This suggests that the $\mathrm{AO}$ adsorbs onto the $\mathrm{ZnO}$, depleting the charge carriers near the surface. This type of response to AO was also noted by Osborne [9]-[11] for the thin $\mathrm{ZnO}$ films from which his sensors were fabricated, except that the response rapidly became non-linear and the resistance (or, rather, conductance in his case) reached an asymptotic value as the sensors became saturated with $\mathrm{AO}$. In the present experiments there was no indication of sensor saturation.

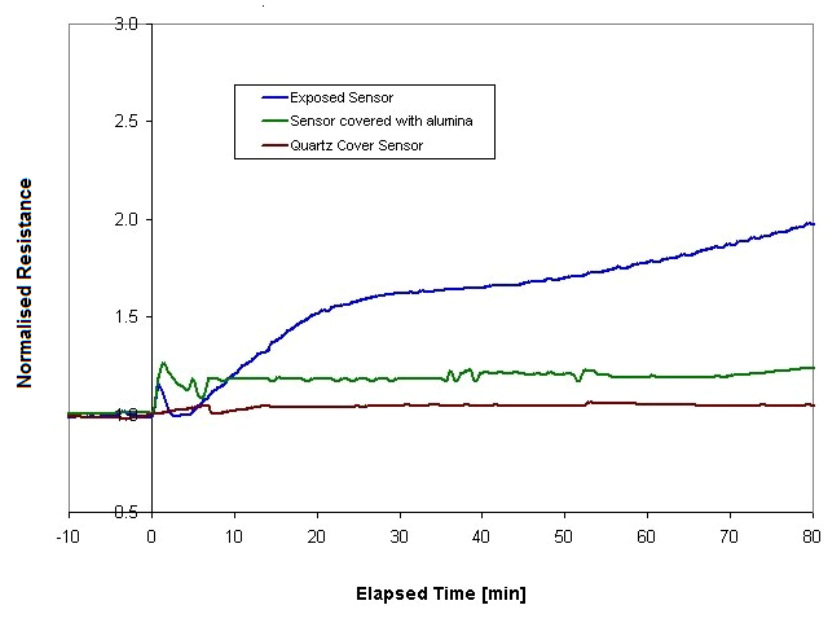

Fig. 3. Variation of normalized resistance against time of AO exposure for a bare (exposed) sensor, a sensor covered with quartz and a sensor covered with alumina.

Evidently the sensors protected from AO exposure did not respond to the background environment in the test chamber or to UV produced by the ATOX facility. The latter observation is somewhat surprising, since zinc oxide is normally considered to be UV photoconductive [20]; in similar control experiments Osborne [9], [11] demonstrated that his thin-film $\mathrm{ZnO}$ sensors, protected from $\mathrm{AO}$ by a thin film of silica, decreased in resistance when exposed to the ATOX-induced UV radiation. The apparent lack of UV-response noted in the present thick film sensors is attributed to the fact that the UV can penetrate only just beneath the surface of the thick film (e.g. Heiland [20] showed that the penetration depth of UV in $\mathrm{ZnO}$ is of the order of $0.1 \mu \mathrm{m}$ ), leaving the majority of the thick film unaffected.

After achieving a fluence of about $10^{19}$ atoms $/ \mathrm{cm}^{2}$ (equivalent to about 9 hours exposure in LEO at an altitude of $330 \mathrm{~km}$ - see Section 1), the sensors were heated under vacuum conditions at $135^{\circ} \mathrm{C}$ overnight (approx. 15 hours) so as to allow the desorption of atomic oxygen. It was found that the resistance returned to a value of the same order of magnitude previously achieved in vacuum conditions before exposure. Subsequent exposures to AO produced similar results to that shown in Fig. 3, indicating that the sensors remained responsive to $\mathrm{AO}$ after regeneration.

\section{IMPEDANCE SPECTROSCOPY EXPERIMENTS}

The purpose of this phase of the work was to foster a better understanding of the conduction mechanisms in the thick film sensors that were under development, as well as to attempt to measure AO fluence by means of Impedance Spectroscopy.

Impedance Spectroscopy (IS) is a technique widely used to characterize sensors, and to understand their electrical behavior [21]. In comparison to measurements of DC resistance its primary advantage is its ability to measure the individual contributions to resistance, namely: grain bulk, grain boundaries, connectors, etc. Impedance $(\mathrm{Z})$ is normally measured by applying an AC voltage over a wide range of frequencies to a sample and measuring the current response. 
Further data processing allows the construction of an equivalent electrical circuit that models the electrical response of the system. The data are usually presented as Nyquist plots in which the negative imaginary component of $Z(=-Z$ ') is plotted against the real component (= Z') over the range of frequencies tested. The appropriate equivalent electrical circuit for the sensor under investigation is then determined by attempting to match the modelled and observed Nyquist plots. Impedance Spectroscopy measurements were carried out in the ATOX facilities at ESTEC whilst the sensors were exposed to AO under similar conditions to those described previously. The IS data were obtained using an Ivium Technologies Compact Stat Electrochemical Interface device. The applied AC potential was $1 \mathrm{~V}$ (below the non-linear threshold, see Section III) and the frequency range was from $4 * 10^{6} \mathrm{~Hz}$ down to $0.1 \mathrm{~Hz}$; the sensor temperature was again held constant at $35^{\circ} \mathrm{C}$.

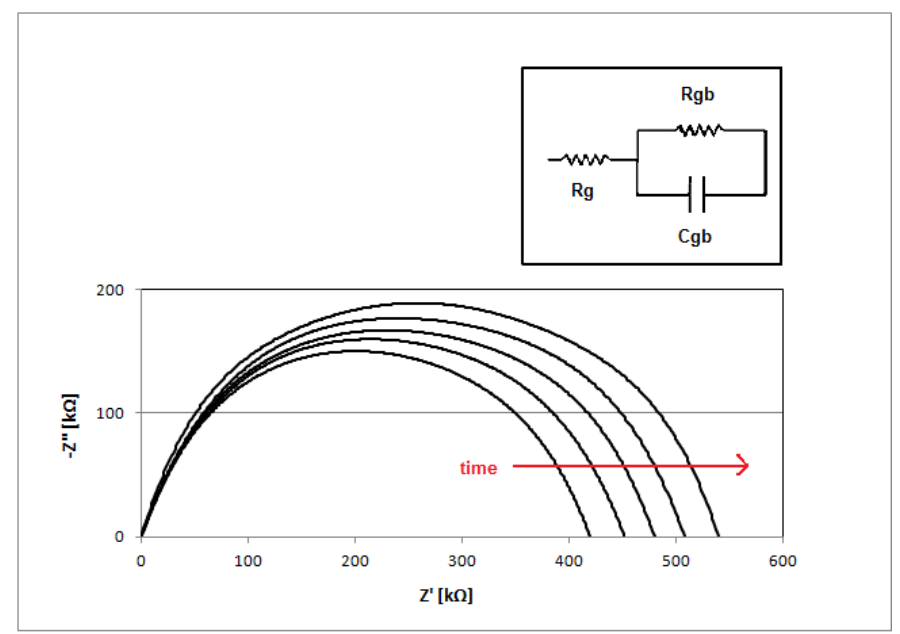

Fig. 4. Nyquist plots of - Z' against Z' obtained by Impedance Spectroscopy, showing the evolution with time of exposure to AO (curves obtained at approximately 30-minute intervals). The inset shows the simplified equivalent electrical circuit used to model the response.

The resultant Nyquist plots obtained for one of the sensors tested are shown in Fig. 4. In this plot the innermost curve corresponds to the sensor initially under vacuum conditions prior to $\mathrm{AO}$ exposure and the other curves indicate the data obtained at approximately half-hourly intervals as the sensor was continually exposed to AO. As, in each case, the Nyquist plots derived from these experiments were approximately semi-circular in shape it was considered that the appropriate equivalent circuit to characterize the response comprised a resistor representing the grain bulk resistance, $\left(\mathrm{R}_{\mathrm{g}}\right)$, in series with a resistor in parallel $\left(\mathrm{R}_{\mathrm{gb}}\right)$ with a capacitor $\left(\mathrm{C}_{\mathrm{gb}}\right)$ to model the grain boundary conductivity [22]. For such a circuit the intercepts with the real (horizontal) axis give the bulk resistance $R_{g}$ and the overall resistance $\left(R_{g}+R_{g b}\right)$; these are obtained at high and low frequencies, respectively. The overall resistance is thus equivalent to the DC resistance of the sensor. The slightly flattened semi-circular shape complicates the interpretation of the Nyquist plots a little and indicates a dispersion of the RC time constant, which is typical of a polycrystalline material with a distribution of interfacial resistance and capacitance [23]. Strictly, in such cases a more complex equivalent circuit that includes a constant phase element is required in order to provide an accurate interpretation of the IS data [24]. However, for the present application the simplified circuit described above was considered sufficient to determine the relative magnitudes of the grain boundary and grain bulk resistances. Unfortunately due to signal noise it was not possible to determine the value of the bulk resistance in these experiments from the high frequency intercept, although clearly it was small compared with the overall resistance. Subsequent IS experiments performed under vacuum conditions at the University of Southampton indicated that grain bulk resistance $\left(\mathrm{R}_{\mathrm{g}}\right)$ of the thick $\mathrm{ZnO}$ films was of order $10^{2}-10^{3} \Omega$, which is of a similar magnitude to those reported by Dhananjay et al. [25] for thin films of $\mathrm{ZnO}$. This, in turn, is 2-3 orders of magnitude less than the total resistance $\left(\mathrm{R}_{\mathrm{g}}+\mathrm{R}_{\mathrm{gb}}\right)$.

Figure 4 shows that total resistance increased with time, as found in the initial DC measurements, whereas the grain bulk resistance did not appear to increase significantly. This suggests that the mechanism causing the increase in total resistance is a depletion of electronic density near the grain boundaries caused by the adsorbed AO.

These total resistances have been re-plotted against time and AO fluence in Fig. 5. This plot indicates that the sensors responded in a linear fashion with time when exposed to a constant flux of AO. Fig. 5 also shows that sensor saturation was not achieved at end of the exposure which, in terms of fluence, was equivalent to that of about one day in LEO at typical ISS altitudes. This fluence is about two to three orders of magnitude greater than that which saturated the thin film $\mathrm{ZnO}$ sensors developed by Osborne $[9,10]$.

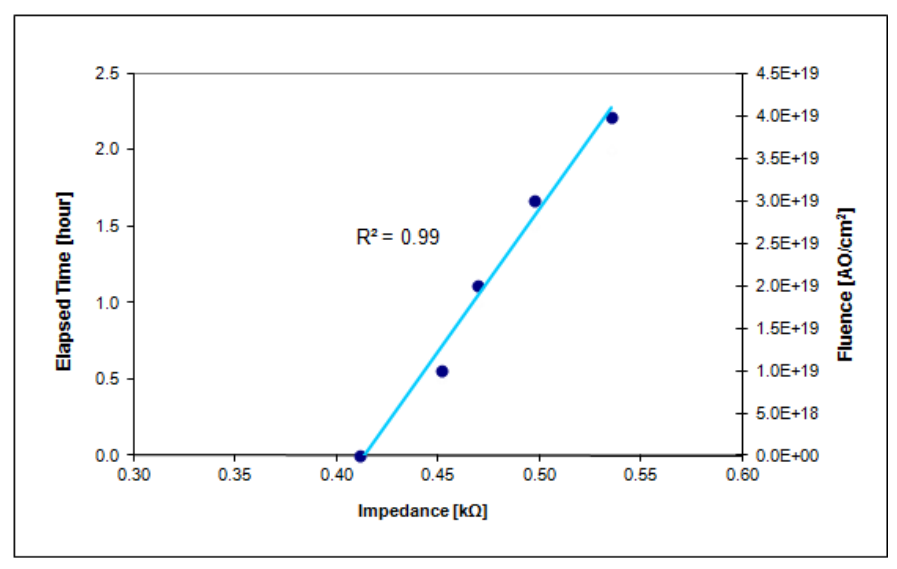

Fig. 5. Total impedance (x-axis) variation with time of AO exposure (y-axis, left) and AO fluence (y-axis, right).

\section{DISCUSSION}

In comparison to the response of Osborne's sensors fabricated from thin, sputtered films of $\mathrm{ZnO}$, the present thick film sensors have exhibited two significant differences in response to AO exposure at typical LEO flux and energy 
levels: the resistance increase appears to be linear with $\mathrm{AO}$ fluence, whereas that of Osborne's sensors was non-linear, and the fluence required to saturate the sensors is at least two orders of magnitude greater for the thick film sensors. In order to attempt to explain these differences a theoretical model of the sensor response has been developed. As will be shown below, these two observations are consistent with the observation that the physical structure of the sensor film is that of a porous material with a large effective surface area, as discussed in Section II.

Osborne [9]-[11], following a theoretical treatment originally proposed by Langmuir [26] and later by Haberreker et al. [27], developed a model for AO adsorption and its effect on the conductance (inverse resistance) of a semi-conductor film. Some of the results from that model will be quoted here, but the reader is referred to [9] for details of the derivation. The model is based on the assumption that there is a competition between atom adsorption on, and de-sorption (or re-emission) from, the surface of the semiconductor material, and that adsorption takes place at surface sites provide by the semiconductor crystal atoms. At any given time, $t$, the concentration of adsorbed atoms (in atoms $\mathrm{cm}^{-2}$, say) is given by

$$
\text { d) } t=\left(\frac{a_{\infty}}{1+\frac{C}{(1-\gamma) F \tau}}\right)\left[\exp \left\{-t\left(\frac{(1-\gamma) F_{1}}{C}\right)\left(+\frac{C}{(1-\gamma) F \tau}\right)\right\}\right]
$$

where the parameters in (1) are defined as follows:

$a_{\infty}$ the maximum possible density of adsorbed atoms (atoms $\mathrm{cm}^{-2}$ )

$C$ density of crystal atoms at the surface of the material (atoms $\mathrm{cm}^{-2}$ )

$\gamma$ scattering coefficient

$F$ atom flux (atoms $\mathrm{cm}^{-2} \mathrm{~s}^{-1}$ )

$\tau$ mean atom residence time on the surface (s).

The last of these determines the rate at which atoms are desorbed or re-emitted, and will depend on the surface temperature as well as the ambient pressure. By dimensional reasoning it becomes clear that the parameter combination

$$
\frac{C}{(1-\gamma) F}=\tau_{0}
$$

represents the time taken for the oncoming flux of atoms to adsorb to and occupy all possible surface sites. Combining equations (1) and (2) we get:

$$
\frac{(q) t}{a_{\infty}}=\left(\frac{1}{1+\frac{\tau_{0}}{\tau}}\right)\left[1-\exp \left\{-\frac{t}{\tau_{0}}\left(1+\frac{\tau_{0}}{\tau}\right)\right\}\right]
$$

which represents the fraction of the maximum possible adsorbed atom density at any given time. Unfortunately neither $\tau$ nor $\tau_{0}$ are known theoretically, nor can they be measured easily by experiment. Nevertheless, we can explore equation (4) parametrically by varying $\tau$ and $\tau_{0}$ and plotting the resultant variation of $a(t) / a_{\infty}$ with time. An example is shown in Fig. 6, in which $a(t) / a_{\infty}$ is plotted against $t / \tau_{0}$ for various values of $\tau_{0} / \tau$.

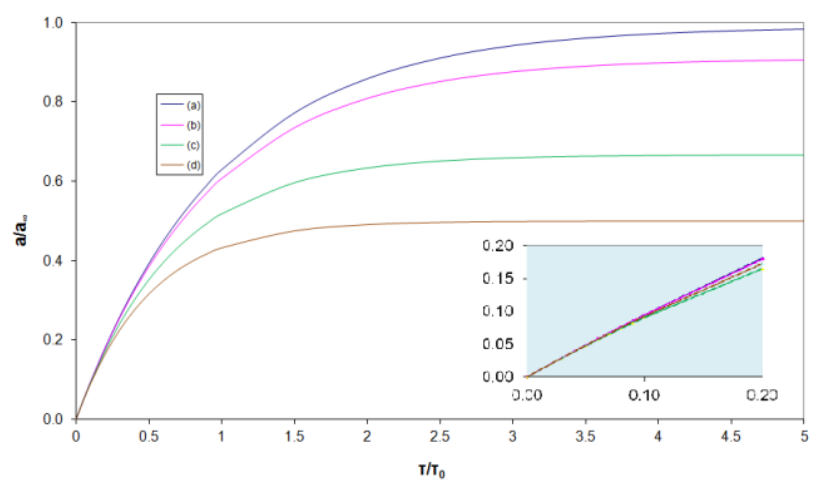

Fig. 6. Modelled variation of the fraction of total surface sites occupied by AO with time. The latter is normalized with $\tau_{0}$, the time taken to occupy all surface sites. (a) $\tau_{0} / \tau=0.01$ (b) $\tau_{0} / \tau=0.1$ (c) $\tau_{0} / \tau=0.5$ (d) $\tau_{0} / \tau=1.0$.

$\tau$ is the AO residence time on the surface. The inset shows the near linear variation in all cases near the origin.

It can be seen that, in all cases, as $t / \tau_{0}$ increases the adsorbed atom fraction $a(t) / a_{\infty}$ asymptotes to a value that depends on the ratio $\tau_{0} / \tau$, reflecting the competition between adsorbtion and re-emission of atoms. At this asymptotic condition the sensors can be considered to be saturated with adsorbed atoms. Note, however, that the atom fraction asymptotes to unity (i.e. all the surface sites are occupied by adsorbed atoms) only when $\tau_{0}<\tau$ (i.e. the time for desorption or re-emission should be significantly larger than that for adsorption). This condition should be satisfied for sensors exposed to LEO AO fluxes, even under vacuum conditions, providing the sensor temperature is not high, as in the present $\mathrm{AO}$ exposure conditions. It should also be noted that, for $\mathrm{t} / \tau_{0} \leq 0.1$, the curves are nearly identical and approximately linear for all the cases shown.

Osborne [9] showed that the conductance of a semiconductor material is reduced (or conversely the resistance is increased) directly in proportion to the surface concentration of $\mathrm{AO}$, assuming the latter deplete the charge carriers in the semiconductor. Hence the resistance of a sensor exposed to AO should increase with time in a similar fashion to that shown in Fig. 6. Note that, in the early stages of exposure (i.e. for $\mathrm{t} / \tau_{0} \leq 0.1$ ), according to the discussion above the increase in resistance of the sensors should be linear and not strongly dependent on the value of the ratio $\tau_{0} / \tau$.

Thus the linear increase (with time) of resistance of the thick film $\mathrm{ZnO}$ sensors in response to a constant flux of $\mathrm{AO}$, as observed in Fig. 5, and the fact that there is no indication of sensor saturation, together imply that $\tau_{0} \gg t$. This in turn suggests that the number of surface sites available for atom adsorption per unit surface area of sensor (the parameter $C$ in equations 1 and 2) is large. This is most probably due to the open, porous nature of the thick film. In contrast, Osborne's dense, thin sputtered films would have offered relatively few 
surface sites for adsorption per unit area; consequently in his case $C$, and consequently $\tau_{0}$, would have been relatively small, indicating that the sensor response to AO exposure would have been non-linear and they would have saturated rather quickly, as observed.

Other forms of $\mathrm{ZnO}$ that also offer a high surface area which have been used for the construction of gas sensors include nano-plates, -wires, -beads and -rods (e.g. [28]-[31]). Also, thick films of $\mathrm{ZnO}$ that have been doped with other metals or metal oxides have been used for gas sensing applications, the doping improving the sensitivity and selectivity to certain gas species (e.g. [32], [33]). To the best of our knowledge none of these relatively novel forms of $\mathrm{ZnO}$ have yet been applied to the sensing of atomic oxygen and this may be a fruitful line of future research.

\section{CONCLUSIONS}

The $\mathrm{ZnO}$ thick film sensors developed in this project have been shown to respond by increasing their resistance when exposed to atomic oxygen. No significant response to ultraviolet radiation has been observed.

Impedance Spectroscopy measurements indicate that the resistance of the grain boundaries of the $\mathrm{ZnO}$ films is $2-3$ orders of magnitude greater than that of the bulk material and therefore dominates the overall (DC) resistance. It has been shown that the overall resistance of the sensors increases when the sensors are exposed to atomic oxygen, whereas that of the grain bulk material remains relatively unaffected. The most likely explanation for this is that the adsorbed species deplete the charge carriers within the sensor material, particularly at the grain boundaries.

When exposed to a constant flux of AO the sensor total resistance increased linearly with time. It is to be expected that the sensors would eventually saturate with adsorbed AO; however, the AO fluence achieved in the experiments reported here (equivalent to that of about one day in LEO at typical ISS altitudes) was evidently insufficient to saturate the sensors. This fluence was more than two orders of magnitude higher than that required to saturate the thin film $\mathrm{ZnO}$ sensors that were developed previously by Osborne. A theoretical model of atom adsorption suggests that this is because the open, porous nature of the thick, screen-printed film sensors offers a large number of surface sites for adsorption in comparison with the thin sputtered film sensors.

It has also been shown that, after exposure to AO, the sensors can be regenerated by heating, the resistance returning to a value of the same order of magnitude as the initial (vacuum) resistance; with repeated subsequent exposures the sensors continue to respond to a flux of AO.

The results indicate that such sensors could be used for long-duration spacecraft missions or in ground-based test facilities in which the total fluence is expected to be high: as soon as the sensors show signs of saturation due to the AO exposure they could be heated to refresh them, after which the AO fluence could then continue to be monitored. However, further work is necessary to improve the robustness of the sensors for such applications. It has been found that this could be achieved by including a suitable binder with the zinc oxide powder and solvent used to fabricate the sensor, but the sensor resistance is increased significantly as a result and the influence of the binder on the sensors' response as yet remains unknown. Also, it would be necessary to calibrate each sensor carefully by first exposing it to $\mathrm{AO}$ at similar flux and energy levels as expected in service.

\section{ACKNOWLEDGEMENTS}

This research was made possible by the support of the European Space Agency under contract number 17762/03/NL/PA. In particular we wish to thank Mark van Eesbeek and Adrian Tighe for their support with the experiments conducted in the ESTEC ATOX test facilities.

We also wish to acknowledge Dr. John Atkinson and his team in the Thick Film Unit of the School of Engineering Sciences, University of Southampton, for the support received to design and manufacture the $\mathrm{ZnO}$ sensors.

\section{REFERENCES}

[1] A. E. Hedin, "Atomic oxygen modelling in the upper atmosphere", Planetary and Space Science, vol. 36, 1988, pp. 907-920.

[2] A. C. Tribble, The Space Environment: Implications for Spacecraft Design, Princeton University Press, 1995.

[3] J. J. Osborne, I. L. Harris, G. T. Roberts, A. R. Chambers, "Satellite and rocket-borne Atomic Oxygen sensor techniques", Review of Scientific Instruments, vol. 72, 2001, pp. 4025-4041.

[4] I. L. Harris, A. R. Chambers, G. T. Roberts, "Results from the Atomic Oxygen experiment on the STRV-1a spacecraft", J. Spacecraft and Rockets, vol. 35 (5), 1998, pp. 647-652.

[5] C. B. White, G. T. Roberts, A. R. Chambers, "Measurement of $5 \mathrm{eV}$ oxygen atoms using carbon-based films: preliminary results", IEEE Sensors Journal, vol. 5 (6), 2005, pp. 1206-1213.

[6] G. Heiland, "Homogeneous semiconducting gas sensors", Sensors and Actuators, vol. 2, 1981, pp. 343-361.

[7] A. I. Livshits, E. E. Gutman, I. A. Myasnikov, V. M. Fursa, V. I. Padeev, Yu. A. Romanovskii, E. I. Kriman, "Atomic-Oxygen analyzer with semiconductor sensors for researching the upper atmosphere of the Earth", Instruments and Experimental Techniques New York, vol. 24, 1981, pp. 754-757.

[8] E. E. Gutman, "Space and Aircraft Sensors", Sensors and Actuators B: Chemical, vol. 18, 1994, pp. 22-27.

[9] J. J. Osborne, "A study of semiconductor-based Atomic Oxygen sensors for ground and satellite applications", $\mathrm{PhD}$ thesis, University of Southampton, 1999.

[10] J. J. Osborne, G. T. Roberts, A. R. Chambers, G. B. Gabriel, "Initial results from ground-based testing of an Atomic Oxygen sensor designed for use in Earth orbit", Review of Scientific Instruments, vol. 70, 1999, pp. 2500-2506.

[11] J. J. Osborne, G. T. Roberts, A. R. Chambers, G. B. Gabriel, "Thin-film semiconductor sensors for hyperthermal Oxygen atoms", Sensors and Actuators B: Chemical, vol. 63, 2000, pp. 55-62.

[12] K. Pitt, Handbook of Thick Film Technology, second ed., Electrochemical Publications, 2005, ISBN 0901150428.

[13] S. Dixit, K. P. Misara, A. Srivastava, A. Srivastava, R. K. Shukla, "An optoelectronic sensor configuration using $\mathrm{ZnO}$ thick film for detection of methanol, Sensors \& Transducers, vol. 82 (8), 2007, pp. 1443-1449.

[14] J. C. Valer, "Development of a reusable Atomic Oxygen sensor using Zinc Oxide thick films", PhD thesis, University of Southampton, 2009.

[15] N. Ohashi, K. Kataoka, T. Ohgaki, T. Miyagi, H. Haneda, K. Morinaga, "Synthesis of Zinc Oxide varistors with a breakdown voltage of three volts using an intergranular glass phase in the Bismuth-Boron-Oxide system", Applied Physics Letters, vol. 83, 2003, pp. 4587.

[16] J. J. Wortman, J. W. Harrison, H. L. Honbarrier, J. Jen, “Thin film oxygen partial pressure sensor", Res. Triangle Inst., Res. Triangle Park, NC, USA, NASA CR-1941, 1972.

[17] K. I. Hagemark, L. C. Chacka, "Electrical transport properties of Zndoped ZnO", Journal of Solid State Chemistry, vol. 15, 1975, pp. 261275. 
[18] G. E. Caledonia, R. H. Krech, B. D. Green, "A high flux source of energetic Oxygen atoms for materials degradation studies", AIAA Journal, vol. 25, 1987, pp. 59-63.

[19] B. Weihs, M. van Eesbeek, "Secondary VUV erosion effects on polymers in the ATOX Atomic Oxygen exposure facility", in Proceedings of the 6th International Symposium on Materials in a Space Environment, ESTEC, Noordwijk, The Netherlands, pp. 277 - 283, 19th -23rd Sep. 1994. ESA SP-368.

[20] G. Heiland, "Photoconductivity of Zinc Oxide", Journal of Physics and Chemistry of Solids, vol. 22, 1961, pp. 227-234.

[21] E. Barsoukov, J. R. Macdonald (Eds), "Impedance Spectroscopy: Theory, Experiment and Applications", second ed., Wiley, ISBN 0-47164749-7, 2005

[22] T. Asokan, R. Freer, "Grain and grain boundary conduction in Zinc Oxide varistors before and after DC degradation, Journal of the European Ceramic Society, vol. 11, 1993, pp. 545-550.

[23] I. D. Raistrick, C. Ho, R. A. Huggins, "Ionic conductivity of some Lithium silicates and aluminosilicates", Journal of the Electrochemical Society, vol. 123, 1976, pp. 1469-1476.

[24] E. J. Abram, D. C. Sinclair, A. R. West, "A strategy for analysis and modelling of impedance spectroscopy data of electroceramics: doped Lanthanum Gallate", Journal of Electroceramics, vol. 10, 2003, pp. 165177.

[25] Dhananjay, J. Nagaraju, S. Krupanidhi, "Investigations on Zinc Oxide thin films grown on $\mathrm{Si}\left(\begin{array}{lll}1 & 0 & 0\end{array}\right)$ by thermal oxidation", Materials Science and Engineering B, vol. 137, 2007, pp. 126-130.

[26] I. Langmuir, "The adsorption of gases on plane surfaces of Glass, Mica and Platinum", Journal of the American Chemical Society, vol. 40, 1918, pp. 1361-1403.

[27] K. Haberreker, E. Mollwo, H. Schreiber, H. Hoinkes, H. Nahr, P. Linder and $\mathrm{H}$. Wilsch, "The ZnO-Crystal as sensitive and selective detector for atomic Hydrogen beams", Nuclear Instruments and Methods, vol. 57, 1967, pp. 22-28.

[28] Z. Jing, J. Zhan, "Fabrication and gas-sensing properties of porous $\mathrm{ZnO}$ nanoplates", Advanced Materials, vol. 20, 2008, pp. 1-5.

[29] S. J. Chang, T. J. Hsuehl, B. R. Huang, et al., "Highly sensitive ZnO nanowire CO sensors with the adsorption of Au nanoparticles", Nanotechnology, vol. 19, 2008, 1775502.

[30] V. R. Shinde, T. P. Gujar, C. D. Lokhande, "Enhanced response of porous $\mathrm{ZnO}$ nanobeads towards LPG: effect of Pd sensitisation", Sensors and Actuators B, vol. 123, 2007, pp. 701-706.

[31] J. X. Wang, L. Vayssieres, et al., Hydrothermally-grown oriented $\mathrm{ZnO}$ nanorod arrays for gas sensing applications", Nanotechnology, vol. 17, 2006, pp. 4995-4998.

[32] D. R. Patil, L. A. Patil, "Room temperature Chlorine gas sensing using surface modified $\mathrm{ZnO}$ thick film resistors", Sensors and Actuators B, vol. 123, 2007, pp. 546-553.

[33] D. R. Patil, L. A. Patil, "Cr2O3-modified $\mathrm{ZnO}$ thick film resistors as LPG sensors", Talanta, vol. 77, 2009, pp. 1409-1411. 